\title{
A projective criterion to the coordinate partitioning method for multibody dynamics
}

\author{
W. Blajer*, W. Schiehlen and W. Schirm, Stuttgart
}

\begin{abstract}
Summary: The paper discusses some developments in the coordinate partitioning method for the dynamic analysis of constrained/closed-loop multibody systems. First, the method is reformulated to a more compact form. Then, a simple and reliable projective criterion for choosing the best coordinates from the redundant ones is proposed, and some advantages are pointed out that may arise in the method by applying inverse kinematics algorithms. Finally, the problem of determination of physical reactions of constraints and closing conditions is discussed. A four-bar linkage mechanism serves for an illustration of some aspects of the paper.
\end{abstract}

\section{Ein Projektionskriterium zur Koordinatenwahl bei Mehrkörpersystemen mit kinematischen Schleifen}

Übersicht: Bei der Simulation der Bewegungsgleichungen von Mehrkörpersystemen mit kinematischen Schleifen in Minimalform stellt sich die Frage nach der Wahl günstiger verallgemeinerter Koordinaten. Hierfür wird ein Projektionskriterium vorgeschlagen, welches die Trennung der systembeschreibenden redundanten Koordinaten in die verallgemeinerten und davon abhängige Koordinaten gestattet. Durch Anwendung eines Verfahrens zur Rückwärtstransformation der kinematischen Beschreibung lassen sich in diesen explizite Schließbedingungen formulieren, was sich bei der Simulation vorteilhaft auswirkt; diese kann ohne Verletzung der Schließbedingungen erfolgen. Es wird auch gezeigt, wie auch bei Verwendung verallgemeinerter Koordinaten physikalisch interpretierbare Reaktionen ermittelt werden können. Als Anwendungsbeispiel dient ein ebenes Viergelenk.

\section{Introduction}

Formulated originally by Wehage and Haug [1], the coordinate partitioning method has been yielded during the last decade a range of general purpose computer codes that are capable of performing dynamic analyses of constrained and/or closed-loop multibody systems. The method treats initially the governing equations of an unconstrained system in the form of differential-algebraic equations (DAEs), composed of the constraint reaction-dependent (in terms of Lagrange multipliers) dynamic equations and the equations of constraints on the system. Then, making use of the fact that the initial ("redundant") coordinates of the system are dependent, a reduced number (equal to the number of degrees of freedom) of independent coordinates is chosen from amoung them. Consequently, the method leads to a minimal set of constraint reaction-free dynamic equations in terms of the independent coordinates, and enables one, in addition, to determine Lagrange multipliers as functions of the current state of the system motion; see also [2]. In this paper a compact and slightly modified formulation of the method is presented and some improvements in its computational efficiency are discussed.

A shortcoming of the coordinate partitioning method is that, for a particular choice of independent coordinates, it may occasionally lead to poorly conditioned matrices or, in the extreme, to singularities. When this occurs, a new set of independent coordinates has to be chosen. A choice is acceptable if a square submatrix of the constraint Jacobian matrix, which relates the dependent coordinates, is nonsingular. However, the method does not originally answer the question which

* On leave from the Department of Mechanics, Technical University of Radom, ul. Malczewskiego 29, 26-600 Radom, Poland 
coordinates are the best to be chosen for the independent ones, which would result in the best conditioned formulation of the method. The comparison of determinant magnitudes of the aforementioned submatrices for possible combinations of independent coordinates can by no means be considered as such a criterion. Therefore, some other related methods generate new (often artificial) independent variables, rather than choose them from the initial ones. By using the zero eigenvalues theorem [3], the singular value decomposition [4]-[6] or the orthogonalization processes [7]-[10], new tangent velocities (the corresponding coordinates may have no physical meaning) are defined, and the final dynamic equations are formulated in terms of these variables. These methods actually lead to the best conditioned dynamic equations only at the point of application. Thus, during the simulation process, they have to be implemented at each step of integration, or occasionally if an additional criterion for acceptability of the current choice of tangent variables is employed. Since the methods are rather computationally expensive, this may be discouraging. Only for some simple and/or evident cases the explicit formulae for the tangent variables can be provided either by inspection [11], [12] or using automatic codes [13].

In the light of the above comments, the coordinate partitioning method in its original (or slightly modified) form can still be attractive, provided that a simple and reliable criterion for the choice of acceptable/best independent coordinates is added. Such a criterion is proposed in this paper. It is also concluded that, as opposed to what has been mentioned in [8], a transition from one set of independent coordinates to another may neither increase the computing time nor propagate integration errors. The method can also be released from the necessity of solving the constraint algebraic equations for the dependent coordinates as function of the independent ones, which commonly have to be introduced at each step of integration and is computationally expensive. Moreover, the paper demonstrates advantages that may arise in the method by applying inverse kinematics algorithms that lead to explicit (though usually recursive) relationships between the dependent and independent coordinates and their derivatives, and that have been developed in the field of robotics [14]. Finally, the problem of determination of physical values of the constraint/closing condition reactions is considered.

\section{Revised formulation of coordinate partitioning}

Consider a mechanical system modelled by $n$ generalized coordinates $\mathbf{q}=\left[q_{1}, \ldots, q_{n}\right]^{T}$ and subject to $m$ holonomic constraints represented by at least twice differentiable functions $\boldsymbol{\Phi}(\mathbf{q}, t)=\left[\Phi_{1}(\mathbf{q}, t), \ldots\right.$, $\left.\Phi_{m}(\mathbf{q}, t)\right]^{T}$. The governing equations of constrained motion of the system can be written in the following DAE form:

$\mathbf{M}(\mathbf{q}, t) \ddot{\mathbf{q}}=\mathbf{h}(\dot{\mathbf{q}}, \mathbf{q}, t)+\mathbf{C}^{T}(\mathbf{q}, t) \lambda$,

$\Phi(\mathbf{q}, t)=\mathbf{0}$,

where $\mathbf{M}$ is the $n \times n$ symmetric positive-definite mass matrix; $\mathbf{h}$ represents the components of applied forces on the system and the gyroscopic terms; $\mathbf{C}=\partial \mathbf{\Phi} / \partial \mathbf{q}$ is the $m \times n$ constraint matrix; and $\lambda=\left[\lambda_{1}, \ldots, \lambda_{m}\right]^{T}$ contains Lagrange multipliers. The constraint equations (2) can be differentiated to:

$\dot{\mathbf{\Phi}}=\mathbf{C}(\mathbf{q}, t) \dot{\mathbf{q}}+\mathbf{a}(\mathbf{q}, t)=\mathbf{0}$,

$\ddot{\boldsymbol{\Phi}}=\mathbf{C}(\mathbf{q}, t) \ddot{\mathbf{q}}+\mathbf{b}(\dot{\mathbf{q}}, \mathbf{q}, t)=\mathbf{0}$,

where $\mathbf{a}=\partial \boldsymbol{\Phi} / \partial t$, and $\mathbf{b}=\dot{\mathbf{C}} \dot{\mathbf{q}}+\dot{\mathbf{a}}$.

The coordinate partitioning method makes use of the fact that only $k=n-m$ from the $n$ initial coordinates $\mathbf{q}$ are independent, denoted $\mathbf{q}_{I}=\left[q_{I 1}, \ldots, q_{I k}\right]^{T}$; the others are refered to as the dependent coordinates in the meaning of this method, $\mathbf{q}_{D}=\left[q_{D 1}, \ldots, q_{D m}\right]^{T}$. Thus, according to the symbolic partitioning,

$\mathbf{q}=\left[\begin{array}{ll}\mathbf{q}_{I}{ }^{T} & \mathbf{q}_{D}{ }^{T}\end{array}\right]^{T}$,

the constraint equations (3) can be rewritten as

$\mathbf{C}_{I}(\mathbf{q}, t) \dot{\mathbf{q}}_{I}+\mathbf{C}_{D}(\mathbf{q}, t) \dot{\mathbf{q}}_{D}+\mathbf{a}(\mathbf{q}, t)=\mathbf{0}$. 
For clarity in the mathematical formulation, this symbolic notation, partitioned relative to independent and dependent coordinates, will be used through the whole paper. In numerical algorithms, however, it is usually more convenient to complete this task by assigning appropriate addresses to the entries of matrices and vectors being partitioned.

If constraints (2) are independent, rank $(\mathbf{C})=m$, there exists at least one set of $\dot{\mathbf{q}}_{D}$ such that the corresponding square submatrix $\mathbf{C}_{D}$ is nonsingular, det $\left(\mathbf{C}_{D}\right) \neq 0$. This enables one to express $\dot{\mathbf{q}}_{D}$ as linear combinations of $\dot{\mathbf{q}}_{I}$, and then $\ddot{\mathbf{q}}_{D}$ as linear combination of $\ddot{\mathbf{q}}_{I}$, i.e.:

$\dot{\mathbf{q}}_{D}=-\mathbf{C}_{D}{ }^{-1}\left(\mathbf{C}_{I} \dot{\mathbf{q}}_{I}+\mathbf{a}\right)=\mathbf{A}(\mathbf{q}, t) \dot{\mathbf{q}}_{I}+\boldsymbol{\eta}(\mathbf{q}, t)$,

$\ddot{\mathbf{q}}_{D}=\mathbf{A}(\mathbf{q}, t) \ddot{\mathbf{q}}_{I}+\xi\left(\dot{\mathbf{q}}_{I}, \mathbf{q}, t\right)$,

where $\mathbf{A}=-\mathbf{C}_{D}{ }^{-1} \mathbf{C}_{I}$ is of dimension $m \times k$; and $\boldsymbol{\eta}=-\mathbf{C}_{D}{ }^{-1} \mathbf{a}$ and $\xi=-\mathbf{C}_{D}{ }^{-1} \mathbf{b}=\dot{\mathbf{A}} \dot{\mathbf{q}}_{I}+\dot{\boldsymbol{\eta}}$ are $m$-vectors. Using (7) and (8), the following interdependences between the initial and independent velocities and accelerations can be introduced:

$\dot{\mathbf{q}}=\left[\begin{array}{c}\dot{\mathbf{q}}_{I} \\ \dot{\mathbf{q}}_{D}\end{array}\right]=\left[\begin{array}{c}\mathbf{I}^{(k)} \\ \mathbf{A}\end{array}\right] \dot{\mathbf{q}}_{I}+\left[\begin{array}{l}0 \\ \eta\end{array}\right]=\mathbf{D}^{T} \dot{\mathbf{q}}_{I}+\left[\begin{array}{l}0 \\ \eta\end{array}\right]$,

$\ddot{\mathbf{q}}=\mathbf{D}^{T} \ddot{\mathbf{q}}_{I}+\left[\begin{array}{l}\mathbf{0} \\ \xi\end{array}\right]$,

where $\mathbf{I}^{(k)}$ denotes the $k \times k$ identity matrix; and $\mathbf{0}$ is the $k$-dimensional null vector.

The $k \times n$ matrix $\mathbf{D}(\mathbf{q}, t)=\left[\mathbf{I}^{(k)} \mathbf{A}^{T}\right]$ has apriori maximal rank, and complements the constraint matrix $\mathbf{C}$ in the $n$-space of the system's configuration, i.e. $\mathbf{D C}^{T}=\left[\mathbf{I}^{(k)}-\left(\mathbf{C}_{D}{ }^{-1} \mathbf{C}_{I}\right)^{T}\right]\left[\mathbf{C}_{I} \mathbf{C}_{D}\right]^{T}$ $=\mathbf{C}_{I}{ }^{T}-\mathbf{C}_{I}{ }^{T} \mathbf{C}_{D}{ }^{-T} \mathbf{C}_{D}{ }^{T}=\mathbf{0}$. Thus, the columns of $\mathbf{D}^{T}$ are (contravariant) components of vectors $\underline{\mathbf{d}}_{j}(j=1, \ldots, k)$ which span the tangent subspace in the $n$-space. On the other hand, the columns of $\mathbf{C}^{T}$ are (covariant) components of constraint vectors $\underline{\mathbf{c}}_{i}(i=1, \ldots, m)$ which span the orthogonal (or constrained) subspace. The tangent and orthogonal subspaces complement each other in the $n$-space, and $\mathbf{D C} \mathbf{C}^{T}=\mathbf{0}$ expresses the orthogonality conditions $\underline{\mathbf{d}}_{j} \cdot \underline{\mathbf{c}}_{i}=0(j=1(1) k ; i=1(1) \mathrm{m})$. Since of linear independence, $\underline{\mathbf{c}}_{1}, \ldots, \underline{\mathbf{c}}_{m}, \underline{\mathbf{d}}_{1}, \ldots, \underline{\mathbf{d}}_{k}$ span a new base $\mathbf{e}_{c d}=\left[\mathbf{e}_{c}^{T} \mathbf{e}_{d}^{T}\right]^{T}$ in the $n$-space, where $\mathbf{e}_{c}=\left[\underline{\mathbf{c}}_{1}, \ldots, \underline{\mathbf{c}}_{m}\right]^{T}$ and $\mathbf{e}_{d}=\left[\underline{\mathbf{d}}_{1}, \ldots, \underline{\mathbf{d}}_{k}\right]^{T}$ are the base vectors of orthogonal and tangent subspaces, respectively. The transformation formula between the (covariant) bases $\mathbf{e}_{c d}$ and $\mathbf{e}_{q}$ is

$\mathbf{e}_{c d}=\left[\begin{array}{l}\mathbf{e}_{c} \\ \mathbf{e}_{d}\end{array}\right]=\left[\begin{array}{c}\mathbf{C} \mathbf{M}^{-1} \\ \mathbf{D}\end{array}\right] \mathbf{e}_{q}=\mathbf{T}_{c d} \mathbf{e}_{q}$,

where $\mathbf{e}_{q}=\left[\underline{\mathbf{k}}_{1}, \ldots, \underline{\mathbf{k}}_{n}\right]^{T}$ are the base vectors spanning the directions of $\mathbf{q}$. The appearance of $\mathbf{M}^{-1}$ in the upper part of $\mathbf{T}_{c d}$ comes evident after a little inspection. Since $\mathbf{C}^{T}$ contains covariant components of the base vectors of the orthogonal subspace, the transformation between the covariant base vectors $\mathbf{e}_{c}$ and $\mathbf{e}_{q}$ requires $\mathbf{C M}^{-1}$. On the other hand, $\mathbf{D}^{T}$ contains contravariant components, and the transformation between $\mathbf{e}_{d}$ and $\mathbf{e}_{q}$ is defined by matrix $\mathbf{D}$. For details see Appendix A or refer to [15, $16]$.

Using the above definitions, the dynamic equations (1) can be projected into the base $\mathbf{e}_{c d}$, which is equivalent to the left-sided premultiplication of these equations by $\mathbf{T}_{c d}$. The tangential projection (into $\mathbf{e}_{d}$ base), after considering (9) and (10), leads to the minimal set of constraint reaction-free (or canonical) dynamic equations in turns of the independent coordinates (see also [17])

$\mathbf{M}_{d}(\mathbf{q}, t) \ddot{\mathbf{q}}_{I}=\mathbf{h}_{d}\left(\dot{\mathbf{q}}_{I}, \mathbf{q}, t\right)$,

where

$\mathbf{M}_{d}=\mathbf{D M D}^{T}=\mathbf{M}_{I I}+\left(\mathbf{M}_{I D} \mathbf{A}\right)^{T}+\mathbf{M}_{I D} \mathbf{A}+\mathbf{A}^{T} \mathbf{M}_{D D} \mathbf{A}$,

$\mathbf{h}_{d}=\mathbf{D}\left(\mathbf{h}-\mathbf{M}\left[\mathbf{0}^{T} \xi^{T}\right]^{T}\right)=\mathbf{h}_{I}+\mathbf{A}^{T} \mathbf{h}_{D}-\left(\mathbf{M}_{I D}+\mathbf{A}^{T} \mathbf{M}_{D D}\right) \boldsymbol{\xi}$,

and $\mathbf{M}_{I I}, \mathbf{M}_{I D}, \mathbf{M}_{D D}, \mathbf{h}_{I}$ and $\mathbf{h}_{D}$ are appropriate submatrices of $\mathbf{M}$ and $\mathbf{h}$ relating $\mathbf{q}_{I}$ and $\mathbf{q}_{D}$, respectively. As $\mathbf{M}$ is the metric tensor matrix of base $\mathbf{e}_{q}$, the metric tensor matrix of base $\mathbf{e}_{c d}$ can be written as

$\mathbf{M}_{c d}=\mathbf{T}_{c d} \mathbf{M T}_{c d}^{T}=\left[\begin{array}{cc}\mathbf{C M}^{-1} \mathbf{C}^{T} & \mathbf{0} \\ \mathbf{0}^{T} & \mathbf{D M D}^{T}\end{array}\right]=\left[\begin{array}{cc}\mathbf{M}_{c} & \mathbf{0} \\ \mathbf{0}^{T} & \mathbf{M}_{d}\end{array}\right]$ 
where $\mathbf{M}_{c}=\mathbf{C M}^{-1} \mathbf{C}^{T}$ and $\mathbf{M}_{d}=\mathbf{D} \mathbf{M D}^{T}$ are the metric tensor matrices of bases $\mathbf{e}_{c}$ and $\mathbf{e}_{d}$, respectively; and 0 is the $k \times m$ null matrix. The above relation, which will be of use in the following, indicates that the orthogonal and tangent subspaces really complement each other in the $n$-space.

By appending $\dot{\mathbf{q}}_{I}=\mathbf{v}_{I}$ to (12), $2 k$ first-order differential equations in $\mathbf{v}_{I}$ and $\mathbf{q}_{I}$ follow. However, since $\mathbf{M}_{d}$ and $\mathbf{h}_{d}$ depend on all initial coordinates $\mathbf{q}$, the constraint (2) have to be solved at each step of integration for $\mathbf{q}_{D}$ as function of the current values of $\boldsymbol{q}_{I}$, and this process is usually computationally expensive. Thus, it is recommendable to integrate (12) together with the kinematic relations (9), and solve the $k+n$ first-order differential equations directly for $\mathbf{v}_{I}\left(=\dot{\mathbf{q}}_{I}\right)$ and $\mathbf{q}$.

\section{Projective criterion for coordinate partitioning}

As an introduction consider the mathematical pendulum shown in Fig. 1. Setting $\boldsymbol{q}=[x, y]^{T}$ as the position of particle $G$, the constraint surface is $\Phi=x^{2}+y^{2}-\varrho^{2}=0$, and the orthogonal and tangent directions are as indicated.

Limiting ourselves to the range $\gamma \in\langle 0, \pi / 2\rangle$, it is evident that the best choice for $\mathbf{q}_{I}$ from $\mathbf{q}$ is: $\mathbf{q}_{I}=y$ for $\gamma \in\langle 0, \pi / 4\rangle ; \mathbf{q}_{I}=x$ for $\gamma \in\langle\pi / 4, \pi / 2\rangle$; and for $\gamma=\pi / 4, x$ and $y$ are of the same priority. Respectively, for $\mathbf{q}_{I}=x$ or $\mathbf{q}_{I}=y$, the coordinate partitioning leads to singularity for the position of $G$ at $A$ or $B$ points. One can also deduce that, for a vector $\mathbf{k}_{i}(i=x$ or $y)$ along the direction of $x$ or $y$, the bigger the projection of $\underline{\mathbf{k}}_{i}$ on the tangent direction the better the corresponding coordinate to be chosen for $\mathbf{q}_{I}$, and the null projection of $\mathbf{k}_{i}$ on $\mathbf{d}$ direction indicates that the corresponding coordinate cannot be set for $\mathbf{q}_{I}$. Quantitatively this can be expressed by $\cos ^{2} \alpha_{i}=\left|\underline{\mathbf{k}}_{i}^{(d)}\right|^{2} /\left|\mathbf{k}_{i}\right|^{2}$ (or $\left.\cos ^{2} \beta_{i}=\left|\underline{\mathbf{k}}_{i}^{(c)}\right|^{2} /\left|\underline{\mathbf{k}}_{i}\right|^{2}\right)$. The bigger $\cos ^{2} \alpha_{i}\left(\right.$ the smaller $\left.\cos ^{2} \beta_{i}\right)$ the better the corresponding coordinate for $\mathbf{q}_{I}$, and $\cos ^{2} \alpha_{i}=0$ (or $\cos ^{2} \beta_{i}=1$ ) denotes the singular choice of $\mathbf{q}_{I}$. For the illustration case, $\cos ^{2} \alpha_{x}=\sin ^{2} \gamma\left(\cos ^{2} \beta_{x}=\cos ^{2} \gamma\right)$, and $\cos ^{2} \alpha_{y}=\cos ^{2} \gamma\left(\cos ^{2} \beta_{y}=\sin ^{2} \gamma\right)$.

The projective criterion for coordinate partitioning proposed in this paper generalizes the above simple geometrical illustrations. In general, however, a system's configuration space is not a Cartesian one but an $n$-dimensional Riemannian space. The norm of a vector in such a space has thus to be redefined according to the vector space geometry. The aspects of contravariant/covariant vector representations are of importance for this definition and for the further base transformations in the $n$-space. For the convenience of the reader, the principles of these concepts are summarized in Appendix A, and one can also refer to [15], [18] and [19]. According to the formulae (A5) in Appendix A, the transformation matrix $\mathbf{T}_{c d}$ defined in (11) is the mapping of the covariant representations $\mathbf{k}_{i}^{*}$ of vectors $\underline{\mathbf{k}}_{i}=\mathbf{k}_{i}^{* T} \mathbf{e}_{q}^{*}(i=1(1) n)$,

$\mathbf{k}_{i}^{*}=\left[0, \ldots, 0, \stackrel{\sqrt{1}, 0, \ldots, 0]^{T}}{i \text {-th position }}\right.$

into $\mathbf{e}_{c d}^{*}$ base, i.e.

$\mathbf{k}_{i}^{*(c d)}=\mathbf{T}_{c d} \mathbf{k}_{i}^{*}=\left[\begin{array}{c}\mathbf{C} \mathbf{M}^{-1} \\ \mathbf{D}\end{array}\right] \mathbf{k}_{i}^{*}$.

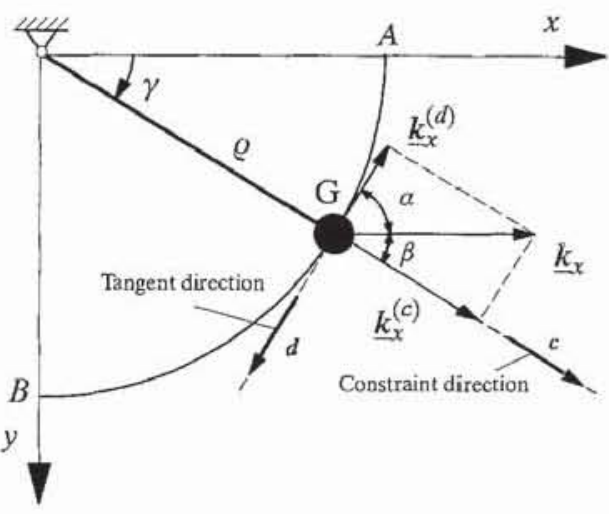

Fig. 1. Projective criterion - concept illustration. Mathematical and physical pendulum, respectively 
The vector $\underline{\mathbf{k}}_{i}$ defined this way can be interpreted as a unit vector along $\dot{q}_{i}$ direction, $\dot{\mathbf{q}} \cdot \underline{\mathbf{k}}_{i}=\dot{\mathbf{q}}^{T} \mathbf{k}_{i}{ }^{*}=\dot{q}_{i}$, and this elucidate its (covariant) representation in (16) (see Appendix A for the meaning of subscripting with *). Then, it comes from (17) that the $i$-th column of $\mathbf{C M}^{-1}$ is the (covariant) representation of $\mathbf{k}_{i}$ in $\mathbf{e}_{c}{ }^{*}$ base, whereas the $i$-th column of $\mathbf{D}$ is the (covariant) representation of $\mathbf{k}_{i}$ in $\mathbf{e}_{d}{ }^{*}$ base. Denoting these representations by $\mathbf{k}_{i}{ }^{*(c)}$ and $\mathbf{k}_{i}{ }^{*(d)}$, respectively, it can be written that:

$\mathbf{C} \mathbf{M}^{-1}=\left[\mathbf{k}_{1}{ }^{*(c)} \mathbf{k}_{2}{ }^{*(c)} \cdots \mathbf{k}_{n}^{*(c)}\right]_{(m \times n)}$,

$\mathbf{D}=\left[\mathbf{k}_{1}{ }^{(d)} \mathbf{k}_{2}{ }^{*(d)} \cdots \mathbf{k}_{n}{ }^{*(d)}\right]_{(k \times n)}$,

i.e. $\mathbf{k}_{i}^{*(c)}$ and $\mathbf{k}_{i}{ }^{*(d)}$ are the $i$-th columns of $\mathbf{C} \mathbf{M}^{-1}$ and $\mathbf{D}$, respectively.

Using (A4) defined in Appendix A, $\left|\mathbf{k}_{i}\right|^{2},\left|\underline{\mathbf{k}}_{i}{ }^{\left({ }^{c}\right)}\right|^{2}$ and $\left|\underline{\mathbf{k}}_{i}^{\left({ }^{(d)}\right.}\right|^{2}$ can be written as follows:

$\left|\underline{\mathbf{k}}_{i}\right|^{2}=\mathbf{k}_{i}{ }^{* T} \mathbf{M}^{-1} \mathbf{k}_{i}{ }^{*}=\mathbf{M}^{-1}(i, i)$,

$\left|\mathbf{k}_{i}^{(c)}\right|^{2}=\left(\mathbf{k}_{i}^{*(c)}\right)^{T} \mathbf{M}_{c}{ }^{-1} \mathbf{k}_{i}^{*(c)}$,

$\left|\underline{\mathbf{k}}_{i}^{(d)}\right|^{2}=\left(\mathbf{k}_{i}^{*(d)}\right)^{T} \mathbf{M}_{d}^{-1} \mathbf{k}_{i}^{*(d)}$,

where $\mathbf{M}^{-1}(i, i)$ is the $i i$-th entry of $\mathbf{M}^{-1}$; and $\mathbf{M}_{c}$ and $\mathbf{M}_{d}$ are defined in (15). Basing on (19), the following generalized formulation of the projective criterion for coordinate partitioning can be introduced:

$\cos ^{2} \alpha_{i}=\frac{\left|\underline{\mathbf{k}}_{i}^{(d)}\right|^{2}}{\left|\underline{\mathbf{k}}_{i}\right|^{2}}=\frac{\left(\mathbf{k}_{i}^{*(d)}\right)^{T} \mathbf{M}_{d}^{-1} \mathbf{k}_{i}^{*(d)}}{\mathbf{M}^{-1}(i, i)}$

$\cos ^{2} \beta_{i}=\frac{\left|\underline{\mathbf{k}}_{i}^{(c)}\right|^{2}}{\left|\underline{\mathbf{k}}_{i}\right|^{2}}=\frac{\left(\mathbf{k}_{i}^{*(c)}\right)^{T} \mathbf{M}_{c}^{-1} \mathbf{k}_{i}^{*(c)}}{\mathbf{M}^{-1}(i, i)}$.

The bigger $\cos ^{2} \alpha_{i}$ (the smaller $\cos ^{2} \beta_{i}$ ) the closer is $\underline{\mathbf{k}}_{i}$ to the tangent hyperplane and the better $q_{i}$ serves as an independent coordinate.

The above formulation appeals directly to the mathematical pendulum illustration introduced at the beginning of this section, and generalizes the concept introduced there to an $n$-dimensional metric space. A crucial role in this formulation plays a redefinition of a vector norm (squared length) by the employment of the metric of the system's configuration space. This enables one to deal automatically with possible different dimensions of $\mathbf{q}$ components. Only in those simple cases (as the mathematical pendulum case) when $\mathbf{M}$ has the nonzero entries solely along its diagonal and all the diagonal elements are the same in both value and dimension, the implementation of metric tensor can be disregarded.

In fact two formulae for the reported criterion have been introduced, (23a) and (23b). Respectively, they express the squared cosines (generalized to the $n$-spaces) of angles between the vector $\underline{\mathbf{k}}_{i}$ and its projections $\underline{\mathbf{k}}_{i}^{(d)}$ and $\underline{\mathbf{k}}_{i}^{(c)}$ into the tangent and orthogonal subspaces. The matrix $\mathbf{M}_{d}$ used in (23 a) is actually the mass matrix of the minimal-dimension dynamic equations (12), and thus is available (more or less explicitly) in its inverted form at each instant of the system motion simulation. The matrix $\mathbf{M}_{c}=\mathbf{C} \mathbf{M}^{-1} \mathbf{C}^{T}$ used in $(23 \mathrm{~b})$ has to be formulated and inverted individually. Therefore, the formulation ( $23 \mathrm{a}$ ) is recommendable for the reported formulation. Using the approach described in [22] where the equations of motion (1) are determined in a recursive way, the formulation (23b) is superior because the matrix $\mathbf{M}_{c}$ is needed at every time step for the determination of the constraint reactions. Furthermore, by using a recursive formalism, the inverted mass matrix $\mathbf{M}^{-1}$ is determined explicitly at every instant of motion. As shown in the following example, this criterion also evaluates the possible sets of differential algebraic equations of motion and therefore serves as a criterion for possible cuts of multibody systems with closed-loops. Especially by using a recursive formalism, the proposed criterion is essential due to the fact that this formalism fails by cutting the loop at a body with neglected mass. This failure cannot be avoided by the reduction to state space form and therefore the choice of a good cut according to the projective criterion is necessary, for details see [23].

For a current set $\mathbf{q}_{I}$, the reported criterion can be applied occasionally to check or redefine the choice for $\mathbf{q}_{I}$ as related those components of $\mathbf{q}$ whose corresponding $\cos ^{2} \alpha_{i}(i=1, \ldots, n)$ have the biggest values. These aspects will be illustrated in more detail in Section 6 , where a four-bar mechanism is analyzed. Here, for tutorial reasons, let us introduce another simple illustration. 
Consider now the pendulum as in Fig. 1 to be a physical pendulum with a body $G$. By setting $\mathbf{q}=[x, y, \gamma]^{T}$, the metric tensor of $\mathbf{e}_{q}$ base is $\mathbf{M}=\operatorname{diag}(m, m, J)$, and $\mathbf{M}^{-1}=\operatorname{diag}\left(\frac{1}{m}, \frac{1}{m}, \frac{1}{J}\right)$; where $m$ is the mass and $J$ the moment of inertia of body $G$. Then, introducing the constraint equations: $\Phi_{1}=x-\varrho \cos \gamma=0$ and $\Phi_{2}=y-\varrho \sin \gamma=0$, the constraint matrix is:

$\mathbf{C}=\left[\begin{array}{ccc}1 & 0 & \varrho \sin \gamma \\ 0 & 1 & -\varrho \cos \gamma\end{array}\right]$

According to the possible choice $\mathbf{q}_{I}=x, \mathbf{q}_{I}=y$ or $\mathbf{q}_{I}=\gamma, \mathbf{D}$ defined in (9) is:

$\mathbf{D}_{x}=\left[1-\frac{\cos \gamma}{\sin \gamma}-\frac{1}{\varrho \sin \gamma}\right]$,

$\mathbf{D}_{y}=\left[\begin{array}{lll}-\frac{\sin \gamma}{\cos \gamma} & 1 & \frac{1}{\varrho \cos \gamma}\end{array}\right]$,

$\mathbf{D}_{\gamma}=\left[\begin{array}{lll}-\varrho \sin \gamma & \varrho \cos \gamma & 1\end{array}\right]$,

and the corresponding matrices $\mathbf{M}_{d}$ are: $\left(\mathbf{M}_{d}\right)_{x}=\left[\frac{m \varrho^{2}+J}{\varrho^{2} \sin ^{2} \gamma}\right] ;\left(\mathbf{M}_{d}\right)_{y}=\left[\frac{m \varrho^{2}+J}{\varrho^{2} \cos ^{2} \gamma}\right] ;$ and $\left(\mathbf{M}_{d}\right)_{\gamma}$ $=m \varrho^{2}+J$. Then, for each possible choice of $\mathbf{q}_{I}$, the criterion ( $23 \mathrm{a}$ ) leads to:

$\cos ^{2} \alpha_{x}=\frac{m \varrho^{2}}{m \varrho^{2}+J} \sin ^{2} \gamma$

$\cos ^{2} \alpha_{y}=\frac{m \varrho^{2}}{m \varrho^{2}+J} \cos ^{2} \gamma$,

$\cos ^{2} \alpha_{y}=\frac{J}{m \varrho^{2}+J}$.

The above illustration shows that, irrespective of the $\mathbf{q}_{I}$ choice, the vectors $\underline{\mathbf{d}}_{i}$ defined by $\mathbf{D}_{i}$ in (25) $(i=x, y$, or $\gamma$ ) span the same direction in the 3-dimensional (Riemannian) space. In general, for each (nonsingular) choice of $\mathbf{q}_{I}, \mathbf{e}_{d}=\left[\underline{d}_{1}, \ldots, \underline{d}_{k}\right]^{T}$ span the same tangent subspace. It is also evident that the entries of $\mathbf{D}_{i}$ in (25) express the same proportions between $\mathbf{k}_{x}{ }^{*(d)}, \mathbf{k}_{y}{ }^{*(d)}$ and $\mathbf{k}_{y}{ }^{*(d)}$ for each choice of $\mathbf{q}_{r}$. However, the comparision of these components directly from (25) is meaningless since the $q$ entries are of different dimensions and physical meaning. Only the involvement of the space metric makes it possible to compare the vector projections as in (26). Note also that, for all possible pendulum configurations, neither $\underline{\mathbf{k}}_{x}, \underline{\mathbf{k}}_{y}$ nor $\underline{\mathbf{k}}_{\gamma}$ project entirely into the tangent subspace, $\cos ^{2} \alpha_{i}<1(i=x, y$, or $\gamma)$. Thus none from $x, y$ and $\gamma$ can be considered as a tangent coordinate.

\section{Application of inverse kinematics algorithms}

The essential shortcoming of the coordinate partitioning method is the necessity of inverting $\mathbf{C}_{D}$ in order to determine $\mathbf{A}=-\mathbf{C}_{D}{ }^{-1} \mathbf{C}_{I}, \boldsymbol{\eta}=\mathbf{C}_{D}{ }^{-1} \mathbf{a}$, and $\boldsymbol{\xi}=\mathbf{C}_{D}{ }^{-1} \mathbf{b}$, required for the formulation of (12). During the simulation process $\mathbf{C}_{D}$ has to be inverted at each step of integration, and this may bring some inefficiency in computations.

In this section advantages are emphasizes that may arise in the coordinate partitioning approach to the dynamic analysis of constrained mechanical systems by adapting special algorithms of inverse kinematics developed in the field of robotics, and of remarkable importance is a technique developed by Woernle [14]. According to this technique, the kinematic chains (closed loops) are parted into two open chains so that to select relations with a reduced number of unknowns. Then, setting some coordinates to be frozen (independent), the recursive relations for the other (dependent) coordinates as function of the frozen ones are found without introducing the constraint equations in the form (2), see also [20], [21], and [22]. These recursive relations are denoted symbolically as

$\mathbf{q}_{D}=\mathbf{g}\left(\mathbf{q}_{I}, t\right)$, 
and are recognized also as closing conditions [24]. In fact, (27) are often quite complex, and the amount of labour required for their derivation depends greatly on the skill of the investigator in using the inverse kinematics procedures. Nevertheless, this initial work pays in the further analysis. The (recursive) relations for (7) and (8) are usually not so laborious to be obtained analytically. They can also be derived by using computer symbolical formalisms like NEWEUL [25], [26]. An example of derivation and application of the recursive formulations for (27), (7) and (8) is demonstrated in Section 6 of this paper, see also [21].

The application of inverse kinematics algorithms benefits in analytical (though recursive) formulae for $\mathbf{g}, \mathbf{A}, \boldsymbol{\eta}$ and $\boldsymbol{\xi}$. This accelerates usually the numerical formulation of the tangent dynamic equations (12), and the final governing equations can be written in the following simplified $2 k$-order form:

$\hat{\mathbf{M}}_{d}\left(\mathbf{q}_{I}\right) \dot{\mathbf{v}}_{I}=\hat{\mathbf{h}}_{d}\left(\mathbf{v}_{I}, \mathbf{q}_{I}, t\right)$

$\dot{\mathbf{q}}_{I}=\mathbf{v}_{I}$

where $\hat{\mathbf{M}}_{d}$ and $\hat{\mathbf{h}}_{d}$ correspond to $\mathbf{M}_{d}$ and $\mathbf{h}_{d}$ defined in (13) and (14) after substituting $\mathbf{q}=\left[\mathbf{q}_{I}{ }^{T}\left(\mathbf{g}^{T}\left(\mathbf{q}_{I}, t\right)\right)\right]^{T}$ and $\dot{\mathbf{q}}=\left[\mathbf{v}_{I}{ }^{T},\left(\mathbf{A}\left(\mathbf{q}_{I}, t\right) \mathbf{v}_{I}+\boldsymbol{\eta}\left(\mathbf{q}_{I}, t\right)\right)^{T}\right]^{T}$, where $\mathbf{g}\left(\mathbf{q}_{I}, t\right), \mathbf{A}\left(\mathbf{q}_{I}, t\right), \boldsymbol{\eta}\left(\mathbf{q}_{I}, t\right)$ and $\xi\left(\mathbf{v}_{I}, \mathbf{q}_{I}, t\right)$ represent the recursive formulae from the inverse kinematics. Note that the closing conditions (27) replace the constraint equations (2), i.e. it can be written

$\hat{\mathbf{\Phi}}(\mathbf{q}, t)=-\mathbf{g}\left(\mathbf{q}_{I}, t\right)+\mathbf{q}_{D}=\mathbf{0}$,

and (7) and (8) stand for $(\hat{\boldsymbol{\Phi}})^{\circ}=\mathbf{0}$ and $(\hat{\boldsymbol{\Phi}})^{*}=\mathbf{0}$. Thus, the solution of (28) is released from the problem of constraint violation. Note also that, as all the entries of $\dot{\mathbf{q}}$ and $\mathbf{q}$ are determined at each step of integration of (28), an eventual transition from one set of $\dot{\mathbf{q}}_{I}$ to another will not yield any inconsistency in the initial value problem of accordingly reformulated governing equations. Obviously, an appropriate number of recursive formulae (27), (7) and (8) for different possible (or all) sets of $\mathbf{q}_{I}$ from q has to be prepared in advance.

\section{Determination of constraint/closing condition reactions}

As previously stated, the closing conditions (27) stand for the artificial constraint equations (29), as compared with the physical ones (2). In the first-order kinematic form, the former are

$(\hat{\mathbf{\Phi}})^{\cdot}=-\mathbf{A} \dot{\mathbf{q}}_{I}-\boldsymbol{\eta}+\dot{\mathbf{q}}_{D}=\mathbf{0}$,

i.e. are identical with the resolved form (7) of (6). The corresponding constraint matrix is

$\hat{\mathbf{C}}=\left[\begin{array}{ll}-\mathbf{A} & \mathbf{I}^{(m)}\end{array}\right]$,

where $\mathbf{I}^{(m)}$ is the $m \times m$ identity matrix. The matrix $\hat{\mathbf{C}}$ defines a new set of constraint vectors $\underline{\hat{\mathbf{c}}}_{i}(i=1(1) \mathrm{m})$, which also span the constrained subspace $\left(\mathbf{D} \hat{\mathbf{C}}^{T}=\mathbf{0}\right)$ but, in general, have no reference with the directions and values of the constraint vectors $\mathbf{c}_{i}(i=1(1) m)$ defined by the constraint matrix $\mathbf{C}$ relating constraints (2). Thus, in the formulation $\hat{\mathbf{C}}^{T} \hat{\lambda}=\mathbf{C}^{T} \boldsymbol{\lambda}$ for the total of closing reactions, $\hat{\lambda}=\left[\hat{\lambda}_{1}, \ldots, \hat{\lambda}_{m}\right]^{T}$ conserves the multipliers which can by no means be identified with the reactions on the system (in the physical sense) due to the closing conditions. In fact, $\lambda=\left[\lambda_{1}, \ldots, \lambda_{m}\right]^{T}$ in the term $C^{T} \lambda$ in (1) should not, in general, be identified with the physical reaction values either. Namely, it can be simply demonstrated that, for the planar mathematical pendulum shown in Fig. 1, by setting the constraint equation $\Phi=x^{2}+y^{2}-\varrho^{2}=0$, the constraint matrix is $\mathbf{C}=\left[\begin{array}{ll}2 x & 2 y\end{array}\right]$, and in the term $\mathbf{C}^{T} \lambda$ neither $\lambda$ conserves the constraint reaction values nor $\mathbf{C}$ is the distribution matrix of the reaction forces.

In the light of these comments, the relation for the total of constraint reactions, which force the system to move consistently with the constraint conditions, can be formulated variously, according to the used formulation of the system constrainment. Nevertheless, since particular constraints on the system have usually their physical interpretation, the physical reactions (forces/moments) of these constraints $\mathbf{f}=\left[f_{1}, \ldots, f_{m}\right]^{T}$ can be usually defined and, for the purpose of the further analysis, one can write

$\mathbf{C}^{T} \lambda=\hat{\mathbf{C}}^{T} \hat{\lambda}=\mathbf{B}^{T} \mathbf{f}$, 
where $\mathbf{B}(\mathbf{q}, t)$ is the $m \times n$ full-rank matrix of distribution of reactions $f_{j}(j=1, \ldots, m)$ along $\mathbf{q}$ directions. The $i j$-th component of $\mathbf{B}^{T}(i=1, \ldots, n ; j=1, \ldots, m)$ expresses the interdependence between the virtual displacement of the system constrained point along the assumed direction of $f_{j}$ and the virtual displacement along $\mathbf{q}_{i}$, whereas the degrees of freedom relating the other $\mathbf{q}$ components are frozen. Thus, B can be obtained by using the well-known virtual work method. For most common constraint types, $\mathbf{B}$ can be obtained automatically by symbolical multibody formalism NEWEUL [25]-[27], and probably by many other symbolical computer formalisms. In principle the vectors $\underline{\mathbf{b}}_{i}(i=1, \ldots, m)$ defined by covariant components as columns of $\mathbf{B}^{T}$, are represented only in the orthogonal subspace, $\mathbf{D B}^{T}=\mathbf{0}$, and can be considered as another set of base vectors of this subspace, $\mathbf{e}_{b}=\left[\underline{\mathbf{b}}_{1}, \ldots, \underline{\mathbf{b}}_{m}\right]^{T}$. Thus, the transformation formula (11) can be extended to

$\mathbf{e}_{x d}=\left[\begin{array}{c}\mathbf{X} \mathbf{M}^{-1} \\ \mathbf{D}\end{array}\right] \mathbf{e}_{q}=\mathbf{T}_{x d} \mathbf{e}_{q}$,

where $x=b, c$ or $\hat{c}$; and $\mathbf{X}=\mathbf{B}, \mathbf{C}$ or $\hat{\mathbf{C}}$, respectively. Note that irrespectively of the possible formulation of the orthogonal subspace base vectors, the tangent projection of the initial dynamic equations (1) leads always to some minimal-dimension dynamic equations as defined in (12). The orthogonal projection, respectively to the choice for $\mathbf{X}$, leads to the determination of $\mathbf{f}, \boldsymbol{\lambda}$ or $\hat{\lambda}$, see e.g. [15], [27]-[29]. Since the physical reaction values are of interest, one can write

$\mathbf{f}=\left(\mathbf{B M}^{-1} \mathbf{B}^{T}\right)^{-1} \mathbf{B}\left(\ddot{\mathbf{q}}-\mathbf{M}^{-1} \mathbf{h}\right)$.

Introducing (10) and making use of the fact that $\mathbf{B D}^{T}=\mathbf{0}$, (34) simplify to

$\mathbf{f}=\left(\mathbf{B M}^{-1} \mathbf{B}^{T}\right)^{-1} \mathbf{B}\left(\left[\begin{array}{l}\mathbf{0} \\ \boldsymbol{\xi}\end{array}\right]-\mathbf{M}^{-1} \mathbf{h}\right)$,

and $\mathbf{f}$ depend on the current values of the state variables of the governing equation (17) or (28), and time.

The scheme (35) requires first the inversion of the $n \times n$ matrix $\mathbf{M}$, and then the formulation and inversion of the $m \times m$ matrix $\mathbf{M}_{b}=\mathbf{B} \mathbf{M}^{-1} \mathbf{B}^{T}$. These operations may be expensive in computations. Therefore another, possibly more effective scheme for the determination of $\mathrm{f}$ is proposed in this paper. Namely, using (1), (11) and (32) one can write

$\mathbf{B}^{T} \mathbf{f}=\left[\begin{array}{c}\mathbf{B} \\ \mathbf{D M}\end{array}\right]^{T}\left[\begin{array}{l}\mathbf{f} \\ \mathbf{0}\end{array}\right]=\left(\mathbf{T}_{b d} \mathbf{M}\right)^{T}\left[\begin{array}{l}\mathbf{f} \\ \mathbf{0}\end{array}\right]=\mathbf{M} \ddot{\mathbf{q}}-\mathbf{h}$,

where $\mathbf{0}$ denotes the $k$-dimensional null vector. Since $\mathbf{T}_{b d} \mathbf{M}$ is invertible in principle, $\mathbf{f}$ can be determined from

$\left[\begin{array}{l}\mathbf{f} \\ \mathbf{0}\end{array}\right]=\left(\mathbf{T}_{b d} \mathbf{M}\right)^{-T}(\mathbf{M} \ddot{\mathbf{q}}-\mathbf{h})$.

Note that the $m$ upper rows of $\left(\mathbf{T}_{b d} \mathbf{M}\right)^{-T}$ can be interpreted as the pseudoinverse of $\mathbf{B}^{T}$.

As compared with (35), the scheme (37) involves only one matrix inversion, the inversion of the $n \times n$ matrix $\left(\mathbf{T}_{b d} \mathbf{M}\right)^{T}$, and $k$ lower rows of this matrix can be retrieved gratuitoulsy from the process of formulation of $\mathbf{M}_{d}$. Then the current values of $\ddot{\mathbf{q}}$ can be determined easily from (10), i.e. $\ddot{\mathbf{q}}=\mathbf{D}^{T} \ddot{\mathbf{q}}_{I}+\left[\begin{array}{ll}0^{T} & \xi^{T}\end{array}\right]^{T}$, where $\ddot{\mathbf{q}}_{I}$ are the current values of right sides of the resolved equations (12), or (28a). Therefore, the scheme (37) can really compete with the scheme (35) as concerned the computational effectiveness. On the other hand, both schemes are equivalent, and in Appendix B it is shown that (37) leads directly to (35).

Finally, the reaction values $\mathbf{f}$ can be determined directly from

$\mathbf{B}^{T} \mathbf{f}=\mathbf{M} \ddot{q}-\mathbf{h}$,

where the current values of the right sides of (38) are calculated as mentioned above, and $m$ independent rows of $\mathbf{B}^{T}$ must be identified in order either to inverte the corresponding square submatrix or, preferably, to use Gauss' elimination method. The numerical aspects of the three mentioned approaches for the determination of $\mathbf{f}$ are analysed in more detail in [27]. 


\section{Example}

Consider the planar four-bar linkage shown in Fig. 2. In order to build an open-loop system, each of the joints $O_{i}(i=1(1) 4)$ can be cut, yielding a one branch or two branch tree, respectively. The coordinates $\mathbf{q}$ of the unconstrained system can also be defined differently. In the example case, the mechanism was cut in joint $O_{4}$ and the relative coordinates $\mathbf{q}=\left[\begin{array}{ll}\alpha_{1} & \alpha_{2} \\ \alpha_{3}\end{array}\right]^{T}$ have been chosen to represent a planar manipulator with the end-effector fixed in point $O_{4}$. The dynamic equations of the system, corresponding to (12), will not be reported here.

As stated in Sections 2 and 4, the system constrainment can be expressed either implicitly by constraint equations (2) or explicitly by closing conditions (27), which yields respective formulations of matrix D defining the tangent subspace. Since the former method is rather a standard one, we will demonstrate in the following only an application of inverse kinematics algorithms leading to recursive relations for the closing conditions. The subsequent derivation for the simple example case bases on the approach given in [14], to where the reader is refered for details. According to the approach, we seperate the mechanism, by cutting in joints $\mathrm{O}_{2}$ and $\mathrm{O}_{4}$, into the lower and upper segments, and the closure condition is

$\overrightarrow{\mathbf{r}}_{\text {lower }} \circ \overrightarrow{\mathbf{r}}_{\text {lower }}-\overrightarrow{\mathbf{r}}_{\text {upper }} \circ \overrightarrow{\mathbf{r}}_{\text {upper }}=0$,

where $\overrightarrow{\mathbf{r}}_{\text {lower }}=\overrightarrow{\mathrm{O}_{1} \mathrm{O}_{4}}-\overrightarrow{\mathrm{O}_{1} \mathrm{O}_{2}}, \overrightarrow{\mathbf{r}_{\text {upper }}}=\overrightarrow{\mathrm{O}_{3} \mathrm{O}_{2}}-\overrightarrow{\mathrm{O}_{3} \mathrm{O}_{4}}$, and $(\overrightarrow{)})$ denotes a vector. Due to the used segmentation, (39) depends on $\alpha_{1}$ and $\alpha_{3}$ (does not depend on $\alpha_{2}$ ), and only one of these coordinates can be chosen for an independent one in the subsequent derivation (the choice $q_{I}=\left[\alpha_{2}\right]$ would require a different segmentation). Here, the relations (27), (9) and (10) are reported only for $\mathbf{q}_{I}=\left[\alpha_{1}\right]$.

Solving (39), one obtaines

$\alpha_{3}= \pm \arccos \left(\frac{l_{0}^{2}+l_{1}^{2}-l_{2}^{2}-l_{3}^{2}-2 l_{0} l_{1} \sin \alpha_{1}}{2 l_{2} l_{3}}\right)$.

The complementary relation for $\alpha_{2}$ is obtained then as suggested in [14] from

$\sin \alpha_{2}=\frac{l_{0} l_{2} \cos \alpha_{1}+l_{0} l_{3} \cos \left(\alpha_{1}+\alpha_{3}\right)+l_{1} l_{3} \sin \alpha_{3}}{l_{0}^{2}+l_{1}^{2}-2 l_{0} l_{1} \sin \alpha_{1}}$,

$\cos \alpha_{2}=\frac{l_{0} l_{2} \sin \alpha_{1}+l_{0} l_{3} \sin \left(\alpha_{1}+\alpha_{3}\right)-l_{1} l_{3} \cos \alpha_{3}-l_{1} l_{2}}{l_{0}^{2}+l_{1}^{2}-2 l_{0} l_{1} \sin \alpha_{1}}$.

The relations (40) and (41) express recursively $\alpha_{3}\left(\alpha_{1}\right)$ and $\alpha_{2}\left(\alpha_{1}\right)$ as defined in (27). Differentiation of these closing conditions leads to:

$\dot{\alpha}_{3}=\frac{l_{0} l_{1} \cos \alpha_{1}}{l_{2} l_{3} \sin \alpha_{3}} \dot{\alpha}_{1}$,

$\dot{\alpha}_{2}=-\frac{l_{0}}{l_{3}} \frac{\cos \left(\alpha_{1}+\alpha_{2}\right)}{\sin \alpha_{3}} \dot{\alpha}_{1}-\dot{\alpha}_{3}$,

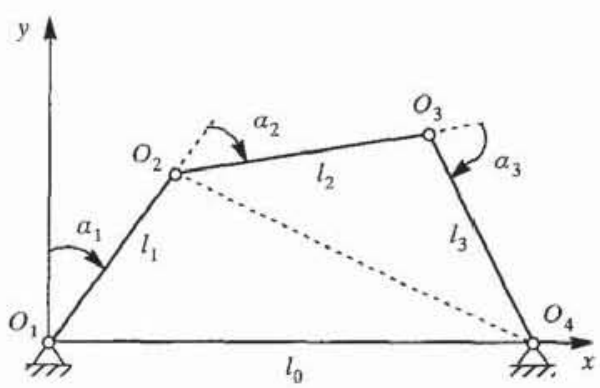

Fig. 2. Four-bar mechanism 


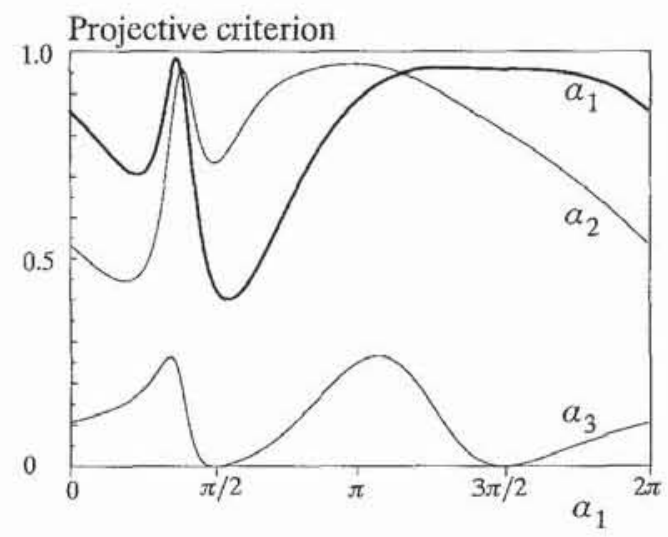

Fig. 3. Projective criterion (four-bar mechanism)

and therefore, the matrix $\mathbf{D}$ defined in (9) can be stated as

$$
\mathbf{D}=\left[1-\frac{l_{0} l_{1} \cos \alpha_{1}+l_{0} l_{2} \cos \left(\alpha_{1}+\alpha_{2}\right)}{l_{2} l_{3} \sin \alpha_{3}} \frac{l_{0} l_{1}}{l_{2} l_{3}} \cdot \frac{\cos \alpha_{1}}{\sin \alpha_{3}}\right] \text {. }
$$

After differentiating (42) the vector $\xi$ introduced in (10) can be formed as seen in [21].

Let us discuss now shortly the problem of the best independent coordinate choice. For particular linkage data, the results obtained by using the projective criterion are shown in Fig. 3. The linkage geometry was set to assure that the choice $q_{I}=\left[\alpha_{1}\right]$ never leads to a singularity, enabling us to plot the results throughout the whole range $\alpha_{1} \in\langle 0,2 \pi\rangle$. As seen, both $\alpha_{1}$ and $\alpha_{2}$ are acceptable choices for $\mathbf{q}_{I}$ in any linkage configuration, none of them, however, can be assigned to be the best independent coordinate over the whole range of $\alpha_{1}$. It is evident, also, that the choice $\mathbf{q}_{I}=\left[\alpha_{3}\right]$ is the worst as leading to singularities at $\alpha_{1}=\pi / 2$ and $\alpha_{1}=3 \pi / 2$, and due to relatively small values of tangent projections.

The numerical simulation can now be carried out in the best independent coordinate according to the projective criterion, i.e. changes between the coordinates $\alpha_{1}$ and $\alpha_{2}$ are necessary to ensure the integration with the best coordinate. That this change can be done without a loss in integration order and stepsize (by using Adams-Bashforth integration code) has been shown in [31].

In comparison with other criteria, i.e. Singular-Value Decomposition (SVD) [4], no difference exist with respect to the singularity points because of their kinematic nature. There are differences, however, in the indication for the best independent coordinate, as the other criterion bases only on the system geometry whereas the projective criterion includes additionally the inertia characteristics. It can be seen that SVD method gives the same results irrespective the changes in the link masses, and the projective criterion is sensitive to these changes. This may be of importance when the inertia characteristics of particular links vary considerably. Another advantage of the proposed criterion lies in its simple geometrical interpretation and comparatively high computational efficiency.

In order to evaluate the reaction forces in joint $O_{4}$, matrix $\mathbf{B}$ is needed. It can be built by using a formalism implemented in NEWEUL and MAPLE [26] as:

$$
\mathbf{B}^{T}=\left[\begin{array}{cc}
0 & -l_{0} \\
-l_{1} \cos \alpha_{1} & -l_{0}+l_{1} \sin \alpha_{1} \\
-l_{1} \cos \alpha_{1}-l_{2} \cos \left(\alpha_{1}+\alpha_{2}\right) & -l_{0}+l_{1} \sin \alpha_{1}+l_{2} \sin \left(\alpha_{1}+\alpha_{2}\right)
\end{array}\right] .
$$

According to (32), the constrainment of the system may affect its dynamics either by $\mathbf{C}^{T} \lambda$ as in (1), by $\mathbf{B}^{T} \mathbf{f}$, where $\mathbf{f}=\left[f_{x}, f_{y}\right]^{T}$ are the reaction forces at joint $O_{4}$ along $x$ and $y$ directions, or by

$$
\hat{\mathbf{C}}^{T} \hat{\lambda}=\left[\begin{array}{cc}
\frac{l_{0} l_{1} \cos \alpha_{1}+l_{0} l_{2} \cos \left(\alpha_{1}+\alpha_{2}\right)}{l_{2} l_{3} \sin \alpha_{3}} & -\frac{l_{0} l_{1}}{l_{2} l_{3}} \cdot \frac{\cos \alpha_{1}}{\sin \alpha_{3}} \\
1 & 0 \\
0 & 1
\end{array}\right] \cdot\left[\begin{array}{c}
\hat{\lambda}_{1} \\
\hat{\lambda}_{2}
\end{array}\right],
$$

which follows from the closing conditions (40)-(42). The reaction forces $\mathbf{f}$ can now be determined using either the scheme (35), (36) or (38). From the computational point of view for the proposed method, scheme (36) is most effective. The solution is found by applying standard solvers for systems 
of linear equations. The direct determination of $\mathbf{f}$ from scheme (38) needs the use of methods for linear least square problems, which are of factor 2 in computation time and therefore inattractive [27]. Finally, scheme (35) proofs to be very efficient only when the dynamic equations are created with the method described in [22], i.e. when the inverted mass matrix $\mathbf{M}^{-1}$ is at hand at every step of integration. Then the explicitly formulated reaction forces can be evaluated parallel to the dynamic simulation without additional matrix inversions.

\section{Conclusions}

The paper motivates to use the coordinate partitioning approach for dynamic analysis of constrained/closed-loop multibody systems. The method is especially useful for those cases when the natural independent coordinates of the systems cannot be defined apriori. A compact mathematical formulation of the method is reported, and the proposed final governing equations (28) release the method from the necessity of solving the algebraic equations of constraints for the dependent coordinates as functions of independent ones, which is an Achilles' heel of the standard approach.

As the method may require occasional redefinition of independent coordinates chosen from the initial (redundant) ones, a conceptually simple and numerically efficient projective criterion for the choice is proposed. The criterion enables one not only to predict and to avoid singularities, but also to define the best set of independent coordinates, resulting in the best conditioned governing equations of the system's constrained motion. Both the geometrical and inertial characteristics of a system are involved for the criterion, the latter being usually omitted in other related methods. Although such a conclusion is usually difficult to state, the reported criterion seems to be numerically very efficient as compared with other methods, and, in fact, it can be implemented only occasionally during the simulation process to check/redefine the best independent coordinates. It is also seen that a transition from one set of $\mathbf{q}_{t}$ to another may neither increase the computing time nor propagate the integration errors.

Furthermore, some advantages that may arise in applications of the method by using inverse kinematics algorithms are indicated. The algorithms lead to explicit formulae between the chosen dependent and independent coordinates, velocities and accelerations. Using the relations, the coordinate partitioning method results in the minimal-set governing equations, which are in principle released from the problem of constraint violation. Again, it is difficult to evaluate benefits of application of the inverse kinematics algorithms as compared to the traditional coordinate partitioning formulation. The inverse formulae, which bave to be obtained analytically either by hand or by symbolical computer manipulations, are usually complex and recursive, and require some skill of the investigator. Also, if changes of independent coordinates are foreseen, a number of sets of the formulae has to be prepared in advance for chosen (or all) combinations for $\mathbf{q}_{I}$ from $\mathbf{q}$. This initial labor will pay in the process of simulation, however, and can be partially automatized.

Finally, three approaches to the determination of physical reactions of constraints/closing conditions are discussed. This may be of importance since the Lagrange multipliers involved in the coordinate partitioning method formulation, and thus able to be determined during the simulation process, have usually no physical meaning.

\section{Appendix A (Vector space notation)}

Consider an $n$-dimensional metric space. A vector $\underline{\mathbf{v}}$ can be represented in this space by its contravariant components $\mathbf{v}=\left[\mathbf{v}_{1}, \ldots, \mathbf{v}_{n}\right]^{T}$ relative the space covariant base $\mathbf{e}=\left[\underline{\mathbf{e}}_{1}, \ldots, \underline{\mathbf{e}}_{n}\right]^{T}$, or by its covariant components $\mathbf{v}^{*}=\left[\mathbf{v}_{1}{ }^{*}, \ldots, \mathbf{v}_{n}{ }^{*}\right]^{T}$ relative the contravariant base $\mathbf{e}^{*}=\left[\underline{\mathbf{e}}_{1}^{*}, \ldots, \underline{\mathbf{e}}_{n}^{*}\right]^{T}$, [30], i.e.

$\underline{\mathbf{v}}=\mathbf{v}^{T} \mathbf{e}=v_{1} \underline{\mathbf{e}}_{1}+v_{2} \underline{\mathbf{e}}_{2}+\ldots+v_{n} \underline{\mathbf{e}}_{n}$

$$
=\mathbf{v}^{* T} \mathbf{e}^{*}=v_{1} \underline{\mathbf{e}}_{1}{ }^{*}+v_{2} \underline{\mathbf{e}}_{2}{ }^{*}+\ldots+v_{n}{ }^{*} \underline{\mathbf{e}}_{n}^{*} \text {. }
$$

With the use of the metric (covariant) tensor matrix (symmetric, positive-definite matrix) of the base $\mathrm{e}$,

$\mathbf{M}=\mathbf{e e}^{T}=\left[\begin{array}{ccc}\underline{\mathbf{e}}_{1} \circ \underline{\mathbf{e}}_{1} & \ldots & \underline{\mathbf{e}}_{1} \circ \underline{\mathbf{e}}_{n} \\ \ldots & \ldots & \ldots \\ \underline{\mathbf{e}}_{n} \circ \underline{\mathbf{e}}_{1} & \ldots & \underline{\mathbf{e}}_{n} \circ \underline{\mathbf{e}}_{n}\end{array}\right]$, 
the interdependences between the contravariant and covariant vector components and base vectors are defined as follows

$\mathbf{v}^{*}=\mathbf{M v} ; \quad \mathbf{v}=\mathbf{M}^{-1} \mathbf{v}^{*} ;$

$\mathbf{e}=\mathbf{M e} \mathbf{e}^{*} ; \quad \mathbf{e}^{*}=\mathbf{M}^{-1} \mathbf{e}$.

The dot product of two vectors $\underline{\mathbf{y}}$ and $\underline{\mathbf{w}}$ can be written in four possible ways:

$\underline{\mathbf{v}} \circ \underline{\mathbf{w}}=\mathbf{v}^{T} \mathbf{M} \mathbf{w}=\mathbf{v}^{T} \mathbf{w}^{*}=\mathbf{v}^{* T} \mathbf{M}^{-1} \mathbf{w}^{*}=\mathbf{v}^{* T} \mathbf{w}$.

When the reference frame changes from the given one to another, denoted ( $\left.{ }^{\wedge}\right)$, the transformation formulae are:

$\hat{\mathbf{e}}=\mathbf{T e} ; \quad \hat{\mathbf{v}}^{*}=\mathbf{T v}^{*} ;$

$\hat{\mathbf{e}}^{*}=\mathbf{T}^{-T} \mathbf{e}^{*} ; \quad \hat{\mathbf{v}}=\mathbf{T}^{-T} \mathbf{v}$,

where $\mathbf{T}$ is the $n \times n$ transformation (invertible) matrix. The metric (covariant) tensor matrix (symmetric, positive-definite matrix) $\hat{\mathbf{M}}$ of the base $\hat{\mathbf{e}}$ is

$\hat{\mathbf{M}}=\hat{\mathbf{e}} \hat{\mathbf{e}}^{T}=\mathbf{T M T}^{T}$.

\section{Appendix B (Equivalence of schemes (35) and (37))}

According to (A6) and with reference to (15), the metric tensor matrix $\mathbf{M}_{b d}$ of the base $\mathbf{e}_{b d}=\left[\underline{\mathbf{b}}_{1}, \ldots, \underline{\mathbf{b}}_{m}, \underline{\mathbf{d}}_{1}, \ldots, \underline{\mathbf{d}}_{k}\right]^{T}$ is

$\mathbf{M}_{b d}=\mathbf{T}_{b d} \mathbf{M T}_{b d}^{T}=\mathbf{T}_{b d}\left(\mathbf{T}_{b d} \mathbf{M}\right)^{T}=\left[\begin{array}{cc}\mathbf{B} \mathbf{M}^{-1} \mathbf{B}^{T} & \mathbf{0} \\ \mathbf{0}^{T} & \mathbf{D} \mathbf{M D}^{T}\end{array}\right]$.

Thus, it is easy to show that

$$
\begin{aligned}
\left(\mathbf{T}_{b d} \mathbf{M}\right)^{-T}=\mathbf{M}_{b d}^{-1} \mathbf{T}_{b d} & =\left[\begin{array}{cc}
\left(\mathbf{B M}^{-1} \mathbf{B}^{T}\right)^{-1} & \mathbf{0} \\
\mathbf{0}^{T} & \left(\mathbf{D M D}^{T}\right)^{-1}
\end{array}\right]\left[\begin{array}{c}
\mathbf{B} \mathbf{M}^{-1} \\
\mathbf{D}
\end{array}\right] \\
& =\left[\begin{array}{cc}
\left(\mathbf{B M}^{-1} \mathbf{B}^{T}\right)^{-1} & \mathbf{B M}^{-1} \\
\left(\mathbf{D} \mathbf{M D}^{T}\right)^{-1} & \mathbf{D}
\end{array}\right] .
\end{aligned}
$$

Substituting (B2) for $\left(\mathbf{T}_{b d} \mathbf{M}\right)^{-T}$ and (10) for $\ddot{\mathbf{q}}$, the scheme (37) reads as follows

$$
\begin{aligned}
{\left[\begin{array}{l}
\mathbf{f} \\
\mathbf{0}
\end{array}\right]=} & {\left[\begin{array}{c}
\left(\mathbf{B M}^{-1} \mathbf{B}^{T}\right)^{-1} \mathbf{B} \mathbf{M}^{-1}\left(\mathbf{M D}^{T} \ddot{\mathbf{q}}_{I}+\mathbf{M}\left[\begin{array}{l}
\mathbf{0} \\
\xi
\end{array}\right]-\mathbf{h}\right) \\
\left(\mathbf{D M D}^{T}\right)^{-1} \mathbf{D}\left(\mathbf{M D}^{T} \ddot{\mathbf{q}}_{I}+\mathbf{M}\left[\begin{array}{l}
\mathbf{0} \\
\xi
\end{array}\right]-\mathbf{h}\right)
\end{array}\right] } \\
= & {\left[\begin{array}{c}
\left.\left(\mathbf{B M}^{-1} \mathbf{B}^{T}\right)^{-1} \mathbf{B}\left(\left[\begin{array}{l}
\mathbf{0} \\
\xi
\end{array}\right]-\mathbf{M}^{-1} \mathbf{h}\right)\right], \\
\mathbf{M}_{d}^{-1}\left(\mathbf{M}_{d} \ddot{\mathbf{q}}_{I}-\mathbf{h}_{d}\right)
\end{array}\right] }
\end{aligned}
$$

where $\mathbf{M}_{d} \ddot{\mathbf{q}}_{I}-\mathbf{h}_{d} \equiv \mathbf{0}$ according to (12).

\section{References}

1. Wehage, R. A.; Haug, E. J.: Generalized coordinate partitioning for dimension reduction in analysis of constrained dynamic systems. Trans. ASME/J. Mech. Design 104 (1982) 247-255

2. Haug, E. J.; Yen, J.: Generalized coordinate partitioning methods for numerical integration of differential-algebraic equations of dynamics. In: Haug, E. J.; Deyo, R. C. (eds.) pp. 97-114. Real-Time Integration Methods for Mechanical System Simulation, NATO ASI Series, Vol. F69, Berlin: Springer 1990 
3. Walton, W. C.; Steeves, E. C.: A new matrix theorem and its application for establishing independent coordinates for complex dynamical systems with constraints. NASA Technical Report TR-326, 1969

4. Mani, N. K.; Haug, E. J.; Atkinson, K. E.: Application of singular value decomposition for analysis of mechanical system dynamics. Trans. ASME/J. Mechanisms, Transmissions and Automation in Design 107 (1985) 82-87

5. Singh, R. P.; Likins, P. W.: Singular value decomposition for constraint dynamical systems. Trans. ASME/J. Appl. Mech. 52 (1985) 943-948

6. Leister, G.: Beschreibung und Simulation von Mehrkörpersystemen mit geschlossenen kinematischen Schleifen. Düsseldorf: VDI-Verlag 1992

7. Kim, S. S.; Vanderploeg, M. J.: QR decomposition for state space representation of constrained mechanical dynamic systems. Trans. ASME/J. Dynamic Systems, Measurement and Control 108 (1986) 183-188

8. Liang, C. G.; Lance, G. M.: A differentiable null space method for constrained dynamic analysis. Trans. ASME/J. Mechanisms, Transmissions and Automation in Design 109 (1987) 405-411

9. Ider, S. K.; Amirouche, F. M. L.: Coordinate reduction in the dynamics of constrained multibody systems - A new approach. Trans. ASME/J. Appl. Mech. 55 (1988) 899-904

10. Agrawal, O. P.; Saigal, S.: Dynamic analysis of multi-body systems using tangent coordinates. Comp. Struct. 31 (1989) 349-355

11. Hemami, H.; Weimer, F. C.: Modelling of nonholonomic dynamic systems with application. Trans. ASME/J. Appl. Mech. 48 (1981) 177-182

12. Blajer, W.; Parczewski, J.: On realization of program constraints. Part II - Practical implications. Trans. ASME/J. Appl. Mech. 56 (1989) 680-684

13. Nikravesh, P. E.: Systematic reduction of multibody equations of motion to a minimal set. Int. J. Non-Linear Mech. 25 (1990) 143-151

14. Woernle, C.: Ein systematisches Verfahren zur Aufstellung der geometrischen Schließbedingungen in kinematischen Schleifen mit Anwendung bei der Rückwärtstransformation für Industrieroboter. Düsseldorf: VDI-Verlag 1988

15. Blajer, W.: Contribution to the projection method of obtaining equations of motion. Mechanics Research Communications 18 (1991) 293-301

16. Blajer, W.: A projection method approach to constrained dynamic analysis. Trans. ASME/J. Appl. Mech. 59 (1992) 643-649

17. Wampler, C.; Buffinton, K.; Shu-hui, J.: Formulation of equations of motion for systems subject to constraints. Trans. ASME/J. Appl. Mech. 52 (1985) 465-470

18. Maisser, P.: A differential-geometric approach to the multibody system dynamics. ZAMM 71 (1991) T116-T119

19. Maisser, P.: Analytische Dynamik von Mehrkörpersystemen. ZAMM 68 (1988) 463-481

20. Eppinger, M.; Kreuzer, E.: Evaluation of methods for solving the inverse kinematics of manipulators. In: Proc. of the 8-th CISM-IFToMM Symp. on Theory and Practice on Robots and Manipulators, Cracow, 1990 (to appear)

21. Blajer, W.; Schiehlen, W.; Schirm, W.: Dynamic analysis of constrained multibody systems using inverse kinematics. Mechanism and Machine Theory, 28 (1993) 397-405

22. Schiehlen, W.; Blajer, W.: Closing conditions and reaction forces of multibody systems. ZAMM 72 (1992) T 45-T 47

23. Schirm, W.: Symbolisch-numerische Behandlung von Mehrkörpersystemen mit kinematischen Schleifen. Düsseldorf: VDI-Verlag 1993

24. Schiehlen, W.: Computational aspects in multibody system dynamics. Computer Methods in Appl. Mech. Eng. 30 (1991) $569-582$

25. Schiehlen, W. (ed.): Multibody systems handbook. Berlin: Springer 1990

26. Kreuzer, E.; Leister, G.: Programmsystem NEWEUL '90. Anleitung AN-23, Stuttgart: Universität, Institut B für Mechanik 1991

27. Schirm, W:: Reaktionskräfte in Mehrkörpersystemen mit kinematischen Schleifen. Zwischenbericht ZB-67, Stuttgart: Universität, Institut B für Mechanik 1992

28. Wittenburg, J.: Dynamics of systems of rigid bodies. Stuttgart: Teubner 1977

29. Schiehlen, W.: Technische Dynamik. Stuttgart: Teubner 1986

30. Sokolnikoff, I. S.: Tensor analysis: Theory and applications. London: John Wiley \& Sons 1962

31. Mandel, H.: Vermeidung von Singularitäten in Mehrkörpersystemen mit geschlossenen kinematischen Schleifen. Studienarbeit Stud-67, Stuttgart: Universität, Institut B für Mechanik 1992

Received September 6, 1993

Dr. W. Blajer

Prof. Dr.-Ing. Dr. h. c. W. Schiehlen

Dr.-Ing. W. Schirm

Institute B of Mechanics

University of Stuttgart

Pfaffenwaldring 9

D-70550 Stuttgart

Germany 